\title{
Síndrome de Mauriac: reporte de un caso
}

\section{Mauriac syndrome: a case report}

\author{
Sergio Eduardo Pérez-Laínez, ${ }^{1}$ Hugo Rueda-Ochoa, ${ }^{1}$ Sergio Deraz-Cabral, ${ }^{1}$ Luis E. Simental-Mendía ${ }^{2}$
}

\begin{abstract}
Resumen
INTRODUCCIÓN: El síndrome de Mauriac es poco frecuente; se caracteriza por retardo del crecimiento, hepatomegalia y obesidad en niños con diabetes mellitus tipo 1.

CASO CLínICO: Niña de 11 años, 4 meses, con diabetes mellitus tipo 1 de 8 años de evolución, tratada con insulina glargina y ultrarrápida. Los padres refieren aumento de volumen abdominal gradual de aproximadamente 3 meses de evolución y mal control glucémico en los últimos 12 meses. Fue llevada al hospital por un cuadro clínico de 17 horas de evolución, caracterizado por polidipsia y poliuria. En la exploración física se encontraron: retardo del crecimiento, retraso en la maduración puberal y hepatomegalia. Los marcadores de hepatitis viral (virus de hepatitis A, B, C, D, Epstein-Barr, citomegalovirus y toxoplasma) o autoinmunitaria (anti-ADN) se reportaron negativos. La biopsia hepática reveló abundante depósito de glucógeno.

CONCLUSIÓN: Si bien el síndrome de Mauriac es raro debe sospecharse en pacientes con diabetes mellitus tipo 1 no controlada, con la finalidad de establecer un diagnóstico y tratamiento oportuno.
\end{abstract}

PALABRAS CLAVE: Síndrome de Mauriac; hepatomegalia; obesidad; diabetes tipo 1; insulina glargina; hepatitis auntoinmune; hiperglucemia.

\section{Abstract}

INTRODUCTION: Mauriac syndrome is an uncommon condition characterized by growth retardation, hepatomegaly and obesity in children with type 1 diabetes.

CASE REPORT: We describe an 11 years old female patient with type 1 diabetes of 8 years duration treated with glargine and rapid-acting insulin. She presented gradually increased abdominal volume of approximately 3 months duration and poor glycemic control over 6 months. In addition, she exhibited polydipsia and polyuria. Physical examination revealed growth retardation, pubertal delay and hepatomegaly. Markers of viral or autoimmune hepatitis were negatives. Finally, liver biopsy reported abundant glycogen deposition.

CONCLUSION: Although the Mauriac syndrome is an unusual condition, it should be suspected in patients with uncontrolled type 1 diabetes in order to establish a timely diagnosis and treatment.

KEY WORDS: Mauriac syndrome; Hepatomegaly; Obesity; Type 1 diabetes; Insulin Glargine; autoimmune hepatitis; Hyperglycemia.

\footnotetext{
${ }^{1}$ Hospital Materno Infantil de la SSA, Durango, Dgo., México.

${ }^{2}$ Unidad de Investigación Biomédica, Delegación Durango, Instituto Mexicano del Seguro Social, México.
}

Recibido: 1 de diciembre 2017

Aceptado: 24 de agosto 2018

Correspondencia

Luis E. Simental Mendía

luis_simental81@hotmail.com

Este artículo debe citarse como Pérez-Laínez SE, et al. Síndrome de Mauriac: reporte de un caso. Acta Pediatr Mex. 2018;39(6):349-354.

\section{INTRODUCCIÓN}

En 1930, Mauriac describió un síndrome infrecuente caracterizado por retardo en el crecimiento, hepatomegalia y obesidad con una distribución peculiar de grasa en niños con diabetes mellitus tipo $1 .^{1}$ La incidencia de este síndrome ha disminuido considerablemente debido a la introducción de la insulina de acción prolongada y, en consecuencia, mejor 
control glucémico. ${ }^{2}$ La mayoría de los casos se manifiesta durante la adolescencia. ${ }^{3}$ Este síndrome se asocia típicamente con retardo en el crecimiento y retraso en la maduración puberal, lo que debe alertar al médico de posible tratamiento inadecuado y la posibilidad de síndrome de Mauriac, que puede revertirse con el control apropiado de la glucemia. ${ }^{4}$ La causa real sigue sin conocerse; se han descrito varios factores relacionados: inadecuada captación y utilización de glucosa en los tejidos, disminución del factor de crecimiento-1 y de hormona de crecimiento, hipercortisolismo, resistencia o defecto de receptores hormonales de membrana celular, deficiencia de insulina, mal control glucémico, enfermedades autoinmunitarias concurrentes, disminución de la ingesta calórica o trastornos de la alimentación (Figura 1). ${ }^{5}$ Las enfermedades autoinmunitarias relacionadas con más frecuencia con el síndrome de Mauriac en pacientes con diabetes mellitus tipo 1 son la enfermedad de Addison, gastritis autoinmunitaria, enfermedad celiaca e hipotiroidismo. ${ }^{6,7}$

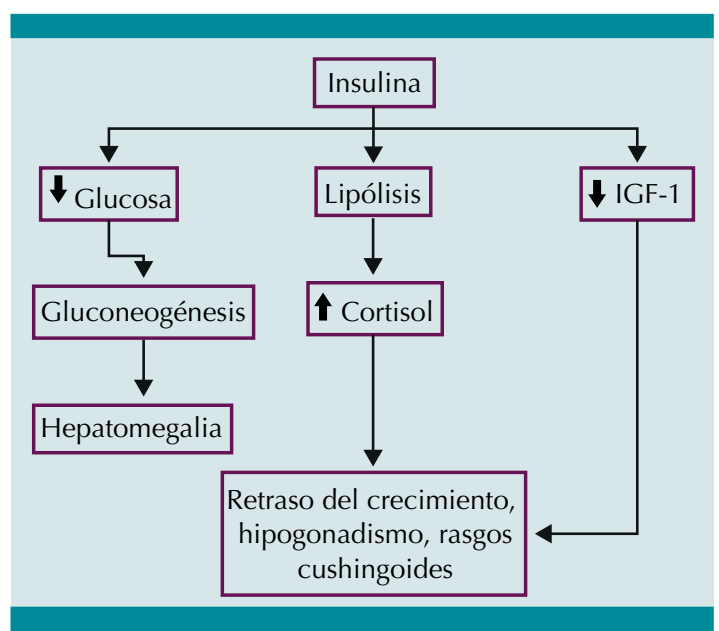

Figura 1. Fisiopatología del síndrome de Mauriac.

\section{CASO CLÍNICO}

Niña de 11 años 4 meses, estudiante del quinto grado de primaria, con antecedente paterno de diabetes mellitus, nivel socioeconómico bajo, talla blanco familiar $1.64 \mathrm{~m}$ (percentil 56), menarquia materna a los 12 años, pubarca paterna desconocida.

La paciente recibió el diagnóstico de diabetes mellitus tipo 1 a los tres años, tratada con insulina glargina $(0.5 \mathrm{UI} / \mathrm{kg} /$ día $)$ e insulina ultrarrápida de manera irregular. El reporte de laboratorio de 6 meses previos a la fecha de consulta fue de: glucosa de $220 \mathrm{mg} / \mathrm{dL}$, urea $21 \mathrm{mg} / \mathrm{dL}$, creatinina $0.5 \mathrm{mg} / \mathrm{dL}$, colesterol total $203 \mathrm{mg} / \mathrm{dL}$, hemoglobina glucosilada (HbA1c) $11.7 \%$ y registro de glucemias capilares preprandiales con promedio de 211 y $267 \mathrm{mg} / \mathrm{dL}$ para el desayuno y la cena, respectivamente. También se recabaron resultados de hemoglobina glucosilada de 1 y 2 años previos con valores de $9.4 \%$ y $8.6 \%$, respectivamente. Además, se refirió aumento gradual del volumen abdominal de aproximadamente 3 meses de evolución. Fue Ilevada a la atención hospitalaria por un cuadro clínico de 17 horas de evolución caracterizado por polidipsia ( $40 \mathrm{~h}$ previas) y poliuria ( $8 \mathrm{~h}$ previas), razón por la que fue hospitalizada para tratamiento y estudio.

En la exploración física se encontró que pesaba $27 \mathrm{~kg}$ (percentil 3), medía $1.40 \mathrm{~m}$ (percentil 10), z talla -1.5 , e IMC de $13.7 \mathrm{~kg} / \mathrm{m}^{2}$, genitales fenotípicamente femeninos, Tanner mamario 1 , Tanner púbico 1, hepatomegalia de aproximadamente $10 \mathrm{~cm}$ por debajo del borde costal, sin esplenomegalia ni ascitis. También se encontró limitación a la extensión de las articulaciones interfalángicas y metacarpofalángicas, sin dolor. Escala de Glasgow 15 puntos. Fondo de ojo con disco óptico y vasos retinianos normales.

Biometría hemática: hemoglobina de $10.2 \mathrm{~g} / \mathrm{dL}$, glucosa plasmática de $270 \mathrm{mg} / \mathrm{dL}$, pruebas de función renal y tiroidea dentro del límite normal y examen general de orina sin alteraciones. Se tomó una gasometría arterial con $\mathrm{pH}$ de 7.42 y $\mathrm{HCO}_{3}$ de 18. Las pruebas de funcionamiento hepático reportaron una elevación importante 
de las transaminasas (alanina aminotransferasa $452 \mathrm{U} / \mathrm{L}$ y aspartato aminotransferasa $102 \mathrm{U} / \mathrm{L}$ ), proteínas totales $5.5 \mathrm{~g} / \mathrm{dL}$, albúmina $2.6 \mathrm{~g} / \mathrm{dL}$, colesterol $299 \mathrm{mg} / \mathrm{dL}$, triglicéridos $234 \mathrm{mg} / \mathrm{dL}$. El valor de la hemoglobina glucosilada fue de $12.4 \%$ y microalbuminuria 17 mg/min.

La edad ósea, utilizando el método de TannerWhitehouse, fue de 8 años para carpo y de 9 años para metacarpos, sin líneas de Park-Harris. Figura 2

Ecografía de hígado y vías biliares: 169 x 136 x $171 \mathrm{~mm}$, volumen aproximado de $2053 \mathrm{~mm}^{3}$. Parénquima ecogénico homogéneo, sin evidencia de lesiones sólidas, quísticas o calcificaciones. Colédoco de $3 \mathrm{~mm}$ de diámetro, vena porta de $5 \mathrm{~mm}$ de diámetro sin ecos en su interior. En la tomografía axial computada se encontró una hepatomegalia importante de origen sin determinar (Figura 3). Por protocolo de estudio se determinó panel de serología viral con reporte negativo de anticuerpos para hepatitis A, B, C

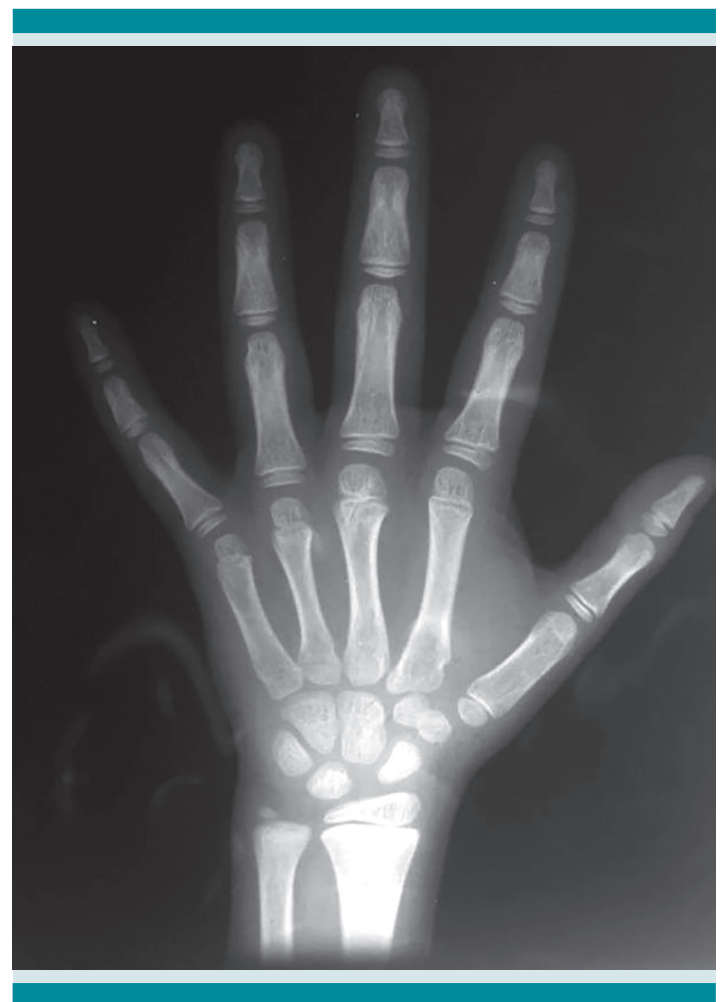

Figura 2. Radiografía posteroanterior de la mano izquierda.
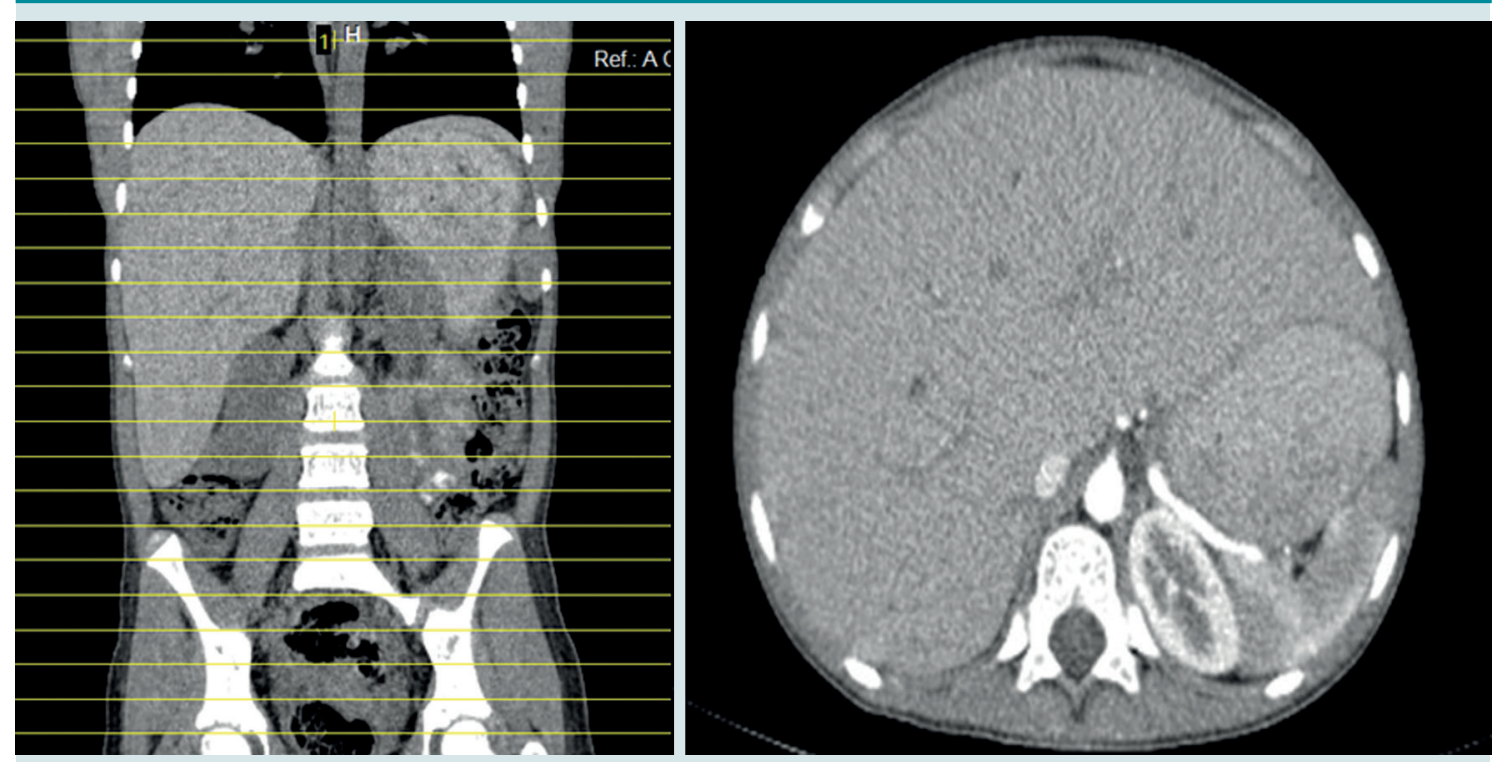

Figura 3. Tomografía axial computada. 
y D. Los resultados para virus anti-Epstein-Barr, anti-citomegalovirus y anti-toxoplasma fueron negativos. También los anticuerpos anti-ADN fueron negativos.

Biopsia hepática: Previa aceptación y firma del consentimiento informado por parte de los padres, se obtuvo una biopsia hepática con aguja tru-cut guiada por ultrasonido. El análisis histopatológico demostró abundantes depósitos de glucógeno con tinción ácido periódico de Schiff (Figura 4). No se realizó microscopía electrónica para visualizar la ultraestructura del depósito.
Durante su estancia hospitalaria se hizo el ajuste de dosis de insulina (glargina de 0.8-1.1 Ul/kg/día y lispro de 0.1 a $0.15 \mathrm{UI} / \mathrm{kg} /$ dosis) y se reforzó el conteo de carbohidratos para mejorar el apego. Las glucosas capilares promedio pre-prandiales del desayuno, comida y cena fueron 156, 121 y $102 \mathrm{mg} / \mathrm{dL}$, respectivamente, y para el posprandio 204, 119 y 122 mg/dL, respectivamente. Cuadro 1

La limitación a la extensión de las articulaciones interfalángicas y metacarpofalángicas no mejoró después del control glucémico durante el internamiento.

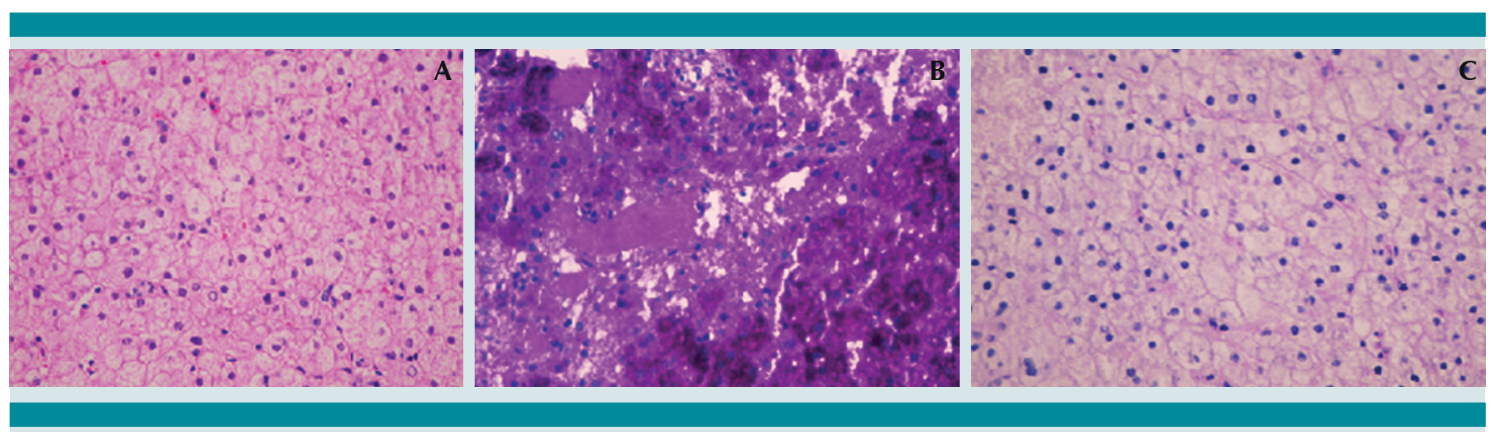

Figura 4. Biopsia hepática: A) Hígado normal, B) Tinción ácido periódico de Schiff, C) Tinción ácido periódico de Schiff-diastasa.

Cuadro 1. Evolución de las pruebas de funcionamiento hepático durante la permanencia hospitalaria

\begin{tabular}{l|c|c|c|} 
& Iniciales & $\mathbf{5}$ días & $\mathbf{1 5} \mathbf{~ d i ́ a s ~}$ \\
\hline $\begin{array}{l}\text { Alanino-amino- } \\
\text { transferasa }\end{array}$ & $452 \mathrm{U} / \mathrm{L}$ & $191 \mathrm{U} / \mathrm{L}$ & $58 \mathrm{U} / \mathrm{L}$ \\
\hline $\begin{array}{l}\text { Aspartato-amino- } \\
\text { transferasa }\end{array}$ & $102 \mathrm{U} / \mathrm{L}$ & $64 \mathrm{U} / \mathrm{L}$ & $39 \mathrm{U} / \mathrm{L}$ \\
\hline Proteínas totales & $5.5 \mathrm{~g} / \mathrm{dL}$ & - & $4.8 \mathrm{gr} / \mathrm{dL}$ \\
\hline Colesterol total & $299 \mathrm{mg} / \mathrm{dL}$ & $204 \mathrm{mg} / \mathrm{dL}$ & $161 \mathrm{mg} / \mathrm{dL}$ \\
\hline Triglicéridos & $234 \mathrm{mg} / \mathrm{dL}$ & $155 \mathrm{mg} / \mathrm{dL}$ & $138 \mathrm{mg} / \mathrm{dL}$ \\
\hline Albúmina & $2.6 \mathrm{~g} / \mathrm{dL}$ & $2.9 \mathrm{~g} / \mathrm{dL}$ & $3.4 \mathrm{~g} / \mathrm{dL}$
\end{tabular}

\section{DISCUSIÓN}

En la actualidad, en todo el mundo se reporta una baja frecuencia del síndrome de Mauriac, ${ }^{2}$ que fue más común en décadas pasadas: Ilegaron a informarse 112 casos en $1942 ;{ }^{\circ}$ sin embargo, tras la incorporación de diferentes tipos de insulina al tratamiento de los pacientes con diabetes mellitus tipo 1, este padecimiento es hoy excepcional. No obstante, en países subdesarrollados, como el nuestro, con escasez de recursos, ligado al descuido por parte de los familiares del paciente, el síndrome de Mauriac podría no ser tan extraño en niños o adolescentes con diabetes mellitus tipo 1 descontrolada, situación que obliga a descartar su diagnóstico por parte del pediatra.

Un signo relevante para el diagnóstico del síndrome de Mauriac es la hepatomegalia. Este hallazgo, en nuestro caso, se confirmó por biopsia hepática que reportó un depósito abundante de 
glucógeno. Al respecto, Mauriac sostenía que la hepatomegalia se debía a un déficit exocrino del páncreas (sobre todo lipasa). ${ }^{1}$ Posteriormente, se sugirió la existencia de una hormona conservadora del metabolismo lipídico denominada "factor lipocaico", cuya carencia producía hiperlipidemia y esteatosis hepática. ${ }^{8}$ Hace poco se reportó un caso de síndrome de Mauriac con una mutación en la subunidad catalítica de la glucógeno fosforilasa cinasa hepática (PHKG2), que actúa de manera dominante inhibiendo la actividad de la glucógeno fosforilasa cinasa, lo que disminuye la glucogenólisis y da como resultado concentraciones elevadas de glucógeno en las células hepáticas. ${ }^{9}$ De todas estas teorías, la más aceptada se relaciona con el depósito de glucógeno, de lípidos o de ambos como consecuencia del mal control metabólico de la diabetes mellitus tipo 1. Esto se sustenta en la regresión de la hepatomegalia cuando el régimen terapéutico es adecuado. ${ }^{10}$ Nosotros corroboramos esta hipótesis con nuestra paciente en quien disminuyó considerablemente la hepatomegalia después de indicar el tratamiento apropiado, lo que es consistente con lo reportado por otros autores. ${ }^{11,12}$

Otro de los signos centrales del síndrome de Mauriac es el retardo en el crecimiento, asociado con frecuencia a la pubertad tardía, hallazgo característico en nuestra paciente. ${ }^{13} \mathrm{Al}$ respecto existen diferentes hipótesis de la explicación de este fenómeno. Mauriac lo atribuía a un trastorno de la función diencefalohipofisaria y luego lo relacionaba con una inestabilidad de la insulina. ${ }^{1}$ Daneman y colaboradores ${ }^{14}$ señalan al déficit de somatotropina como factor relacionado con el retardo en el crecimiento. En vista de que en estos pacientes se ha reportado un aumento de la hormona del crecimiento ante la disminución de somatomedina, algunos autores consideran que se debe a una disfunción hepática. ${ }^{15}$ Otros autores consideran la posibilidad de que exista un factor inhibidor de la somatomedina responsable de la baja talla de estos pacientes. ${ }^{14}$ El retraso puberal puede explicarse porque que las concentraciones elevadas de los productos finales de glicación avanzada suprimen la activación de los pulsos de la hormona liberadora de gonadotropina. ${ }^{13}$

Se han descrito dos variantes del síndrome de Mauriac basadas en la presencia o ausencia de obesidad. ${ }^{2}$ Esto coincide con el reporte de nuestra paciente, quien no tenía obesidad ni facies cushinoide. Esta discrepancia podría explicarse por el relativo corto tiempo de evolución del descontrol metabólico, que fue menor de un año, en comparación con otros casos en donde se reportan hasta 10 años con diabetes mellitus tipo 1 no controlada. ${ }^{5}$

La limitación a la movilidad articular también es un síntoma que puede concurrir con el síndrome de Mauriac. Esta alteración puede afectar significativamente los movimientos finos en niños con diabetes mellitus tipo 1. Esto es indicativo de mal control glucémico e, incluso, puede considerarse marcador del desarrollo de complicaciones microvasculares de la diabetes mellitus tipo $1 .{ }^{16}$

El control metabólico adecuado y sostenido en el tratamiento de la diabetes mellitus tipo 1 constituye aún un gran desafío para el pediatra porque el nivel de educación, la estructura familiar y la situación económica del paciente, o bien la disponibilidad de insumos necesarios para su cuidado, interfieren directamente en el tratamiento adecuado y el autocuidado que la enfermedad requiere; situaciones que estuvieron relacionadas con el caso de nuestro paciente. Por lo tanto, se recomienda que la atención sea multidisciplinaria, incluido el apoyo psicológico, para tratar de mejorar el apego al tratamiento.

\section{CONCLUSIÓN}

Paciente pediátrica femenina, con diabetes mellitus tipo 1 y síndrome de Mauriac asociado con mal control glucémico, retardo en el crecimiento, retraso puberal y hepatomegalia. Si bien 
la presentación de este síndrome es excepcional, en la actualidad este reporte invita a sospechar su existencia en pacientes con diabetes mellitus tipo 1 no controlada, en quienes es necesario buscar intencionadamente los signos característicos con la finalidad de establecer el diagnóstico y tratamiento oportunos.

\section{REFERENCIAS}

1. Manrique-Hurtad H. Síndrome de Mauriac. Rev Soc Peru Med Interna. 2011;24(3):146-8.

2. Patidar PP, et al. A rare case of Mauriac syndrome. Indian J Endocrinol Metab. 2012;16(3):486-87. 10.4103/22308210.95759

3. Mauriac P. Gros ventre, hepatomegalie, troubles de croissance chez les enfants diabetiques traites depuis plusiers annee par l'insuline. Gaz Hebd Med Bordeaux. 1930;26:402-10.

4. Kim MS, Quintos JB. Mauriac syndrome: Growth failure and type 1 diabetes mellitus. Pediatr Endocrinol Rev. 2008;5:989-93.

5. Madhu SV, et al. Mauriac syndrome: A rare complication of type 1 diabetes mellitus. Indian J Endocrinol Metab. 2013;17(4):764-65. doi: [10.4103/2230-8210.113780]

6. Hunger-Battefeld W, et al. Prevalence of polyglandular autoimmune syndrome in patients with diabetes mellitus type 1. Med Klin (Munich) 2009;104:183-91. doi: 10.1007/ s00063-009-1030-x.

7. Ferry RJ Jr. Management of pediatric obesity and diabetes. New York City: Humana Press, 2011;383-5.

8. Suárez R, Padrón R. Síndrome de Mauriac. Revista Cubana de Pediatría. 1976; 48:571

9. MacDonald MJ, et al. Discovery of a Genetic Metabolic Cause for Mauriac Syndrome in Type 1 Diabetes. Diabetes. 2016;65(7):2051-9. doi: 10.2337/db16-0099.

10. Farrell M, Bucuvalas J. Systemic disease and the liver. Liver disease in children. 2 nd ed. Ch. 38. Philadelphia: Lippincott Williams and Wilkins; 2001;897-927.

11. Lee RG, Bode HH. Stunted growth and hepatomegaly in diabetes mellitus. J Pediatr 1977; 91:82-4.

12. Rosenfeld R, Spigelblatt L, Chicoine R. False positive sweat test, malnutrition, and the Mauriac syndrome. J Pediatr. 1979;94(2):240-2.

13. Chowdhury S. Puberty and type 1 diabetes. Indian J Endocrinol Metab. 2015;19(Suppl 1): S51-4. doi: 10.4103/22308210.155402.

14. Daneman D, et al. Progressive retinopathy with improved control in diabetic dwarfism (Mauriac's syndrome). Diabetes Care. 1981;4(3):360-5.

15. Garcia DJ, Castillo A. Síndrome de Mauriac: Experiencia en el Hospital Infantil Dr. Robert Reid Cabral. Acta Médica Dominicana. 1986;8(5):193-5.

16. Nagesh VS, Kalra S. Type 1 diabetes: Syndromes in resourcechallenged settings. J Pak Med Assoc. 2015;65(6):681-5.

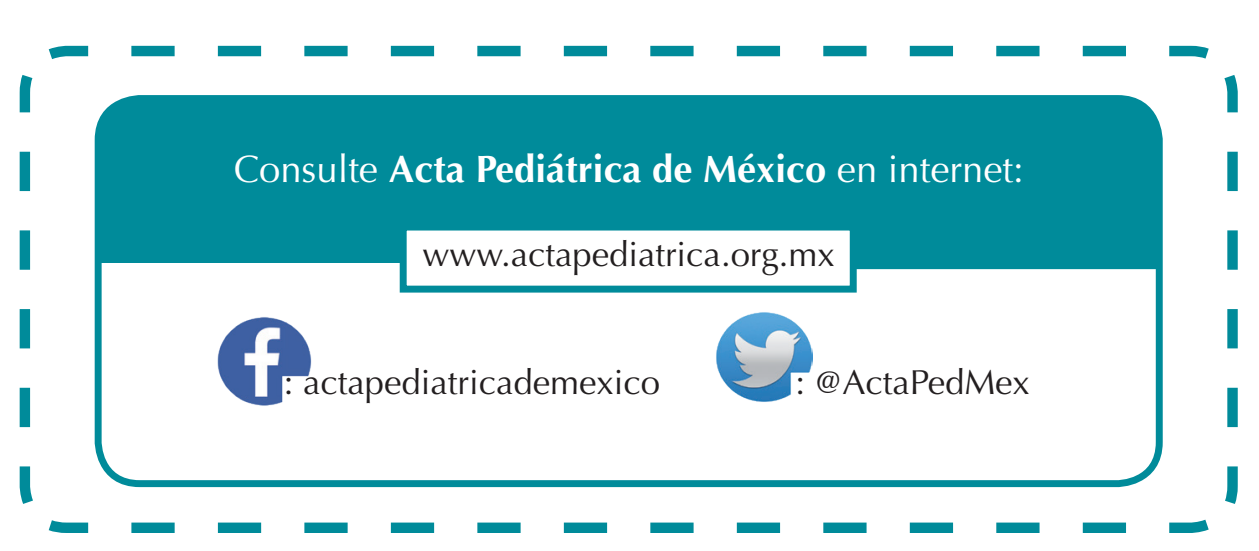

